\title{
Exame ortopédico, com e sem anestesia geral, de cães com luxação patelar medial
}

[Orthopedic exam differences in awake and anaesthetized dogs with medial patellar luxation]

\author{
M.L. Figueiredo ${ }^{1}$, C.E.S.Silva ${ }^{2}$, T.H.T. Fernandes ${ }^{1}$, R. Chioratto ${ }^{3}$, E.A. Tudury ${ }^{3}$ \\ ${ }^{1}$ Aluno de pós-graduação - Universidade Federal Rural de Pernambuco - UFRPE - Recife, PE \\ ${ }^{2}$ Residente - Hospital Veterinário da UFRPE - Recife, PE \\ ${ }^{3}$ Universidade Federal Rural de Pernambuco - UFRPE - Recife, PE
}

\begin{abstract}
RESUMO
Compararam-se os achados musculotendinosos e osteoarticulares encontrados em cães com luxação patelar medial, por meio do exame ortopédico realizado com e sem anestesia geral. Para tal, foram utilizados 11 joelhos de 10 cães, sem distinção de sexo, idade e raça, os quais apresentaram luxação patelar medial dos graus II, III e IV, diagnosticada clinicamente. O exame ortopédico específico da articulação do joelho foi realizado previamente à cirurgia, com o animal sem anestesia, e, no dia da cirurgia, com o cão já anestesiado. Verificou-se, quanto aos achados osteoarticulares, que não houve diferenças. Já em relação aos achados musculotendinosos, houve diferença entre as avaliações do movimento de gaveta e dos músculos sartório e retofemoral.
\end{abstract}

Palavras-chave: joelho, luxação de patela, exame clínico, anormalidades musculo-esqueléticas

\begin{abstract}
This study aimed to compare the osteoarticular and musculotendineal findings in dogs with medial patellar luxation by orthopedic examination performed with and without general anesthesia. For this we used 11 knees of 10 dogs without distinction of sex, age and race, which had medial patellar luxation in grades II, III and IV, diagnosed clinically. The specific orthopedic examination of the knee joint was performed prior to surgery with the non-anaesthetised dog, and on the day of surgery with the same patient already anesthetized. Regarding the osteoarticular results, no differences were found. In relation to the musculotendineal results, differences were observed between the assessments of the drawer movement and sartorius and rectum femoral muscle tension.
\end{abstract}

Keywords: stifle, patella luxation, clinical examination, muscle-skeletal abnormalities

\section{INTRODUÇÃO}

A luxação patelar medial é uma desordem de desenvolvimento que ocorre em cães de raças pequenas, podendo ser causada por traumas em qualquer raça. Geralmente se encontra entre 75 e $80 \%$ dos casos de luxação patelar, em que o envolvimento bilateral dos membros é de 20 a 25\% (Piras, 2011). Dependendo das alterações anatômicas existentes, a luxação medial é classificada em quatro graus, de acordo com o sistema de Putnam (Ferguson, 1997).

Recebido em 1 de março de 2012

Aceito em 1 de outubro de 2012

E-mail: marcellalf@gmail.com
As anormalidades musculoesqueléticas associadas à luxação medial da patela incluem: coxa vara, rotação medial e encurvamento lateral do terço distal do fêmur, genu varum, deslocamento medial do grupo quadríceps, sulco troclear raso com borda medial hipoplásica ou ausente, encurvamento e rotação medial da tíbia com desvio medial de sua crista, displasia da epífise distal do fêmur (côndilo medial hipoplásico), instabilidade rotacional da articulação do joelho e doença articular degenerativa (Maria et al., 2001; Piras, 2011). Segundo Wangdee et al. (2006), o músculo 
sartório sofre alterações estruturais, tornando-se mais tenso e, consequentemente, exercendo tração medial na patela.

Um exame físico cuidadoso é necessário para caracterizar presença de instabilidade patelofemoral, assim como para descartar a possibilidade de ruptura de ligamento cruzado (Vasseur, 2003). O membro deve ser estendido, rotacionado interna e externamente, enquanto se tenta empurrar a patela para medial e lateral em relação ao sulco troclear. Deve-se observar também a presença de crepitação; posição da tuberosidade tibial; incapacidade de redução da patela; profundidade do sulco troclear; localização da patela; contraturas musculares (sartório, retofemoral e quadríceps); incapacidade de estender o membro e presença ou ausência de movimentos de gaveta, pois cada um destes achados afeta os tipos de procedimentos corretivos a serem escolhidos para o reparo cirúrgico (Piermattei et al., 2009; Tudury et al., 2011).

O joelho sempre deve ser examinado quanto à instabilidade decorrente de ruptura do ligamento cruzado (Piermattei et al., 2009). Um resultado falso-negativo pode ocorrer quando o animal é avaliado sem sedação ou anestesia (Hulse, 1995; Slocum e Slocum, 1998a; Johnson e Hulse, 2002; Muzzi et al., 2003), pois o relaxamento permite uma favorável manipulação da articulação sem resistência (Roush, 2001).

Segundo Mann et al. (1988), a fim de se obter uma medida acurada de amplitude total de movimento das articulações, é recomendado anestesiar o animal para excluir restrição consciente dele provocada por dor patológica. Já Jaegger et al. (2002) citam não haver diferenças significativas na realização da goniometria em cães com e sem anestesia.

Objetivou-se neste trabalho comparar os achados musculotendinosos e osteoarticulares encontrados no exame ortopédico, com e sem anestesia geral, de cães com luxação patelar medial.

\section{MATERIAL E MÉTODOS}

Foram avaliados 11 joelhos de cães da rotina do Hospital Escola, sem distinção de sexo, idade e raça, que apresentavam luxação patelar medial de graus II, III e IV. Após o exame clínico, realizou-se exame ortopédico específico de ambos os joelhos, com o animal em decúbito lateral, por meio do qual foi avaliada a existência de: instabilidade medial ou lateral de patela, arrasamento de sulco troclear, desvio de crista tibial, irredutibilidade patelar, crepitação articular, movimento de gaveta cranial e caudal, teste de compressão tibial positivo, crepitação, contraturas musculares (músculos retofemoral e sartório) e deslocamento do grupo muscular quadríceps, conforme Piermattei et al. (2009) e Tudury et al. (2011).

O exame ortopédico específico da articulação do joelho foi realizado previamente à cirurgia, com o animal sem anestesia, e, no dia da cirurgia, após a estabilização anestésica (Tab. 1). Uma comparação entre os resultados desses dois exames foi realizada para identificar diferenças nessas duas situações.

O protocolo anestésico utilizado para a cirurgia e segundo exame ortopédico de cada paciente consistiu de medicação pré-anestésica acepromazina (Acepran 0,2\%, Aventis, São Paulo-SP) $0,2 \%$ (0,05-0,11 mg/kg intramuscular), indução com propofol (Pronest 200mg injetável, Meizler, São Paulo-SP) (4-6mg/kg endovenoso), anestesia epidural com bupivacaína (Neocaina $0,5 \%$, Cristália, Itapira-SP) $0,5 \%(1 \mathrm{~mL} / 4,5 \mathrm{Kg})$ e manutenção com isofluorano (Isofluorano, Cristália, Itapira-SP). Para analgesia préemptiva, foram utilizados tramadol (Tramal 50, Carlo Erba S/A) (2mg/kg subcutâneo) e meloxican (Maxicam 0,2\%, Ouro Fino, São Paulo-SP) (0,2mg/kg subcutâneo). 
Tabela 1. Resultados do exame ortopédico, sem anestesia e com anestesia, realizado nos cães com luxação patelar medial

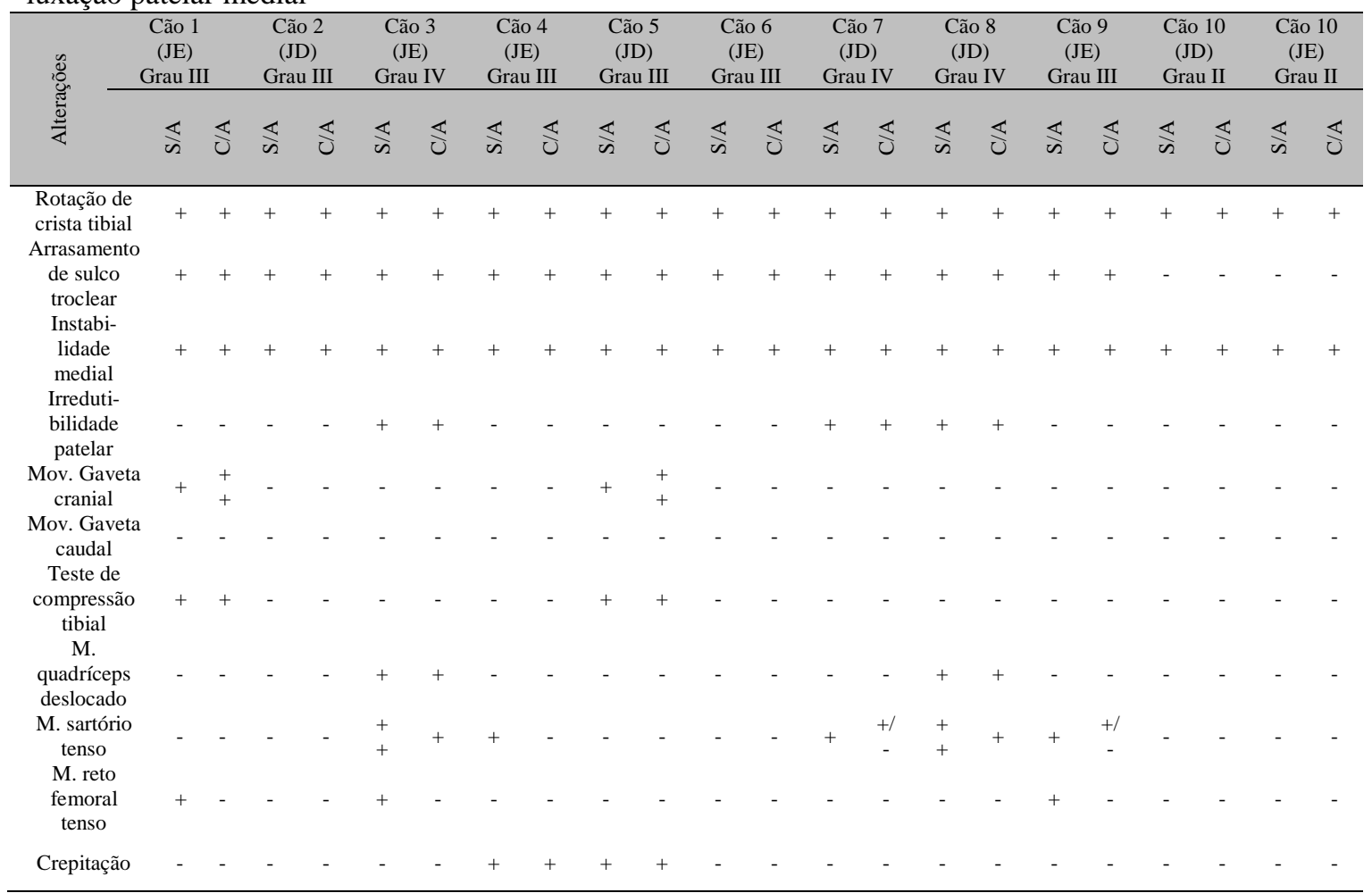

(+) com alteração; (-) sem alteração; (++) muito alterado; (+/-) pouco alterado; (C/A) com anestesia; (S/A) sem anestesia; (JD) joelho direito; (JE) joelho esquerdo.

\section{RESULTADOS E DISCUSSÃO}

Quanto aos achados osteoarticulares: rotação de crista tibial, arrasamento de sulco troclear e instabilidade medial, não houve diferenças nas avaliações efetuadas antes e depois da anestesia. Já em relação aos achados musculotendinosos dos 11 joelhos avaliados, cinco $(3,4,7,8$ e 9$)$ apresentaram o músculo sartório tenso no primeiro exame, porém, com a anestesia, essa alteração muscular diminuiu nos cães 3, 7, 8 e 9, enquanto no cão 4 desapareceu.

Ainda entre esse grupo de animais, que apresentou contraturas musculares, apenas em três $(1,3$ e 9$)$ dos seis animais, foi constatada, no exame sem anestesia, tensão no músculo retofemoral, tensão esta que não foi mais palpável com o animal anestesiado. A tensão constatada no exame com o animal sem anestesia pode ter sido causada pela apreensão ou pela dor que o animal estava sentindo enquanto estava sendo examinado, o que corrobora com Slocum e Slocum (1998) e Muzzi et al. (2003), quando citam que os pacientes podem se apresentar apreensivos, tensos e sentir dor durante o exame, levando à contração da musculatura do membro afetado.

Em relação ao deslocamento do músculo quadríceps, não houve diferença entre os dois exames. Os animais que apresentavam esse deslocamento muscular possuíam luxação patelar medial de grau IV com o músculo quadríceps bastante contraído. Segundo Read (1999), o grau IV pode desencadear deslocamento interno do grupo muscular quadríceps, com ou sem contratura muscular impedindo uma amplitude normal de movimento da articulação do joelho.

Não é um fato isolado que, para se obter resultados confiáveis nos exames ortopédico ou radiográfico, em várias afeções articulares, deve-se realizá-los com o animal sedado ou anestesiado (Mann et al., 1988; Lewis et al., 1992; Slocum e Slocum, 1998; Johnson e Hulse, 2002; Muzzi et al., 2003; Souza e Tudury, 2003; Genevois et al., 2006; Malm et al., 2007), 
concordando esta recomendação com os achados constatados nesta pesquisa quanto às estruturas musculotendíneas.

A incógnita surge do fato de que, quando o animal está acordado, existe uma tensão muscular e ligamentar fisiológica em estação. Ao ser anestesiado, essa tensão fisiológica ou patológica deixará de existir junto com a tensão por medo ou por dor, o que poderá influenciar no pós-operatório do animal, pois esta tensão irá voltar quando o animal acordar da anestesia, podendo ser responsável por recidivas da luxação patelar. Situação semelhante a essa pode ser encontrada em oftalmologia no caso de entrópio reativo, cuja avaliação para correção cirúrgica deve ser realizada antes de qualquer procedimento anestésico, por causa do relaxamento palpebral (Gelatt, 2003).

Dos 10 animais com luxação medial de patela, dois (1 e 5) apresentavam ruptura do ligamento cruzado cranial, a qual foi diagnosticada no primeiro exame. No segundo, já com o animal anestesiado, a realização do movimento de gaveta cranial foi muito mais perceptível, estando de acordo com Hulse (1995), Slocum e Slocum (1998); Johnson e Hulse (2002) e Muzzi et al. (2003), quando citam que, para uma melhor avaliação da integridade desse ligamento, o animal deve estar sedado ou anestesiado.

Segundo Slocum e Slocum (1998), Piermattei et al. (2009), Piras (2011) e Tudury et al. (2011), para correção cirúrgica da luxação medial de patela, devem-se associar técnicas de reconstrução óssea (trocleoplastia e transposição de crista tibial) e de tecido mole (imbricação lateral, suturas antirrotacionais, desmotomia medial, liberação do grupo quadríceps, desinserção dorsal do músculo sartório e transposição do tendão proximal do músculo retofemoral) a fim de se ter o alinhamento do mecanismo extensor e a estabilização da patela na tróclea femoral. Para tanto, é preciso a realização de um exame ortopédico minucioso e confiável para detectar as reais anormalidades musculoesqueléticas a serem corrigidas em cada paciente.

Considerando-se que há mudanças nos achados musculotendinosos em relação aos exames com e sem anestesia, a questão é saber qual a melhor situação para se realizar o exame ortopédico, que irá determinar qual correção cirúrgica será necessária. Já em relação às alterações ósseas, conclui-se que não há diferença quanto aos achados dos exames em um ou outro estado orgânico. Pesquisas futuras poderão elucidar em qual estado de consciência e de relaxamento muscular o animal deverá estar durante o exame ortopédico para se ter maior eficiência e menor número de recidivas no tratamento cirúrgico de cães com luxação patelar medial.

\section{AGRADECIMENTOS}

Ao $\mathrm{CNPq}$, pela bolsa oferecida, que ajudou na realização deste trabalho.

\section{REFERÊNCIAS}

FERGUSON, J. Patellar luxation in the dog and cat. In Practice. v.19, p.174-184, 1997.

GELATT, K.N. Doenças e cirurgia das pálpebras do cão. IN:_. Manual de oftalmologia veterinária. São Paulo: Manole, 2003. cap.3, p.47-72.

GENEVOIS, J.P.; CHANOIT, G.; CAROZZO, C. et al. Influence os anaesthesia on canine hip dysplasia score. J. Vet. Med., v.53, p.415-417, 2006.

HULSE, D.A. The stifle joint. IN: OLMSTEAD, M.L. Small Animal Orthopedics. ST. Louis: Mosby, 1995. cap.18, p.395-416.

JAEGGER， G.; MARCELLIN-LITTLE，D.J.; LEVINE, D. Reliability of goniometry in Labrador Retriever. Am. J. Vet. Res., v.63, p.979986, 2002.

JOHNSON, A.L.; HULSE, D.A. Fundamentals of orthopedic surgery and fracture management. IN: FOSSUM, T.W. Small Animal Surgery. 2.ed. ST. Louis: Mosby, 2002. cap. 33, p.821-900.

LEWIS, D.D.; McCARTHY, R.J.; PECHMAN, R.D. Diagnosis of common developmental orthopedic conditions in canine pediatric patients. Compend. Contin. Educ. Pract. Vet., v.14, p.287-301, 1992.

MALM, S.; STRANDBERG, E.; DANELL, B. et al. Impact of sedation method on the diagnosis of hip end elbom dysplasia in Swdish dogs. Prev. Vet. Med., v.78, p.196-209, 2007. 
MANN, F.A.; WAGNER-MANN, C.; TANGNER, C.H. Manual goniometric measurement of the canine pelvic limb. J. Am. Anim. Hosp. Assoc., v.24, p.189-194, 1988.

MARIA, P.P.; PADILHA-FILHO, J.G.; ALMEIDA, T.L. Luxação medial de patela em cães - revisão. Clin. Vet., v.34, p.25-32, 2001.

MUZZI, L.A.L.; REZENDE, C.M.F.; MUZZI, R.A.L. et al. Ruptura do ligamento cruzado cranial em cães: fisiopatogenia e diagnóstico. Clin. Vet., v.46, p.32-42, 2003.

PIERMATTEI, D.L.; FLO, G.L; DECAMP, C.E. A articulação do joelho. In:_. Brinker, Piermattei e Flo, ortopedia e tratamento de fraturas de pequenos animais, 4.ed., São Paulo: Manole, 2009. p.637-717.

PIRAS, A. Radiographs: what projections are indicated. In: ESVOT, 2011. Lyon. Anais... Lyon: veterinary campus of Lyon, 2011. p.8-11.

READ, R.A. Opciones racionales de tratamiento de la luxación interna de rótula. Waltham Focus, n.4, p.25-31, 1999.

ROUSH, J. K. Hind limb lameness in the mature dog, Veterinary Clinics of North America: Small Animal Practice, v.31, n.1, 2001.
SLOCUM, B.; SLOCUM, T.D. Knee. IN: BOJRAB, M.J. Current techniques in small animal surgery. 4.ed. Baltimore: Williams\&Wilkins, 1998. Cap. 62, p.1187-1244.

SOUZA, A.F.A.; TUDURY, E.A. Displasia coxofemoral: diagnóstico clínico e radiográfico revisão. Clín. Vet., n.47, p.54-66, 2003.

TUDURY, E.A.; FIGUEIREDO, M.L.; ARAÚJO, F.P. et al. Desinserção proximal do músculo sartório na correção da luxação patelar medial graus III e IV, em cães. Arq. Bras. Med. Vet. Zootec., v.63, p.254-257, 2011.

VASSEUR, P.B. Stifle joint. In: SLATTER, D. Textbook of small animal surgery. 3.ed. Philadelphia: Saunders, 2003. cap.147, p.20902133.

WANGDEE, C.; CHUTHATEP, S.; SOONTORNVIPART, K. et al. The structural study of the sartorius of medial patellar luxated dogs. IN: Annual congress of veterinary science, 2006, Chulalongkorn. Proceedings Ann. Con. Vet. Sci. Chula. Meeting/27-28 April, pag.56, 2006. 